\title{
Imaging by Atomic Force Microscopy of the Plasma Membrane of Prestin-Transfected Chinese Hamster Ovary Cells
}

\author{
Michio Murakoshi, ${ }^{1}$ Takashi Gomi, ${ }^{1}$ Koji Iida, ${ }^{1}$ Shun Kumano, ${ }^{1}$ Kouhei Tsumoto, ${ }^{2}$ \\ Izumi Kumagai, ${ }^{3}$ Katsuhisa Ikeda, ${ }^{4}$ Toshimitsu Kobayashi, ${ }^{5}$ and Hiroshi Wada ${ }^{1}$ \\ ${ }^{1}$ Department of Bioengineering and Robotics, Tohoku University, 6-6-01 Aoba-yama, Sendai 980-8579, Japan \\ ${ }^{2}$ Department of Medical Genome Sciences, Graduate School of Frontier Sciences, The University of Tokyo, \\ 5-1-5 Kashiwanoha, Kashiwa 277-8652, Japan \\ ${ }^{3}$ Department of Biomolecular Engineering, Tohoku University, 6-6-11 Aoba-yama, Sendai 980-8579, Japan \\ ${ }^{4}$ Department of Otorhinolaryngology, Juntendo University School of Medicine, 2-1-1 Hongo, Tokyo 113-8421, Japan \\ ${ }^{5}$ Department of Otorhinolaryngology-Head and Neck Surgery, Tohoku University Graduate School of Medicine, \\ 1-1 Seiryo-machi, Sendai 980-8574, Japan
}

Received: 16 November 2005; Accepted: 26 April 2006; Online publication: 8 June 2006

\begin{abstract}
The high sensitivity of mammalian hearing is achieved by amplification of the motion of the cochlear partition. This cochlear amplification is thought to be generated by the elongation and contraction of outer hair cells (OHCs) in response to acoustical stimulation. This motility is made possible by a membrane protein embedded in the lateral membrane of OHCs. Although a fructose transporter, GLUT-5, was initially proposed to be this protein, a later study identified the gene of the motor protein distributed throughout the OHC plasma membrane. This protein has been named "prestin." However, although previous morphological studies by electron microscopy and atomic force microscopy (AFM) found the lateral wall of OHCs to be covered with 10 -nm particles, believed to be motor proteins, it is unknown whether such particles consist only of prestin or are a complex of GLUT-5 and prestin molecules. To determine if the 10-nm particles are indeed constituted only of prestin, plasma membranes of prestin-transfected and untransfected Chinese hamster ovary (CHO) cells, which do not express GLUT-5, were observed by AFM. First, the cells attached to a substrate
\end{abstract}

Correspondence to: Hiroshi Wada - Department of Bioengineering and Robotics - Tohoku University - 6-6-01 Aoba-yama, Sendai 9808579, Japan. Telephone: +81-22-7956938; fax: +81-22-7956939; email: wada@cc.mech.tohoku.ac.jp were sonicated so that only the plasma membrane remained on the substrate. The cytoplasmic face of the cell was observed by the tapping mode of the AFM in liquid. As a result, particle-like structures were recognized on the plasma membranes of both the prestintransfected and untransfected $\mathrm{CHO}$ cells. Comparison of the difference in the frequency distribution of these structures between those two cells showed approximately $75 \%$ of the particle-like structures with a diameter of 8-12 $\mathrm{nm}$ in the prestin-transfected $\mathrm{CHO}$ cells to be possibly constituted only by prestin molecules. Our data suggest that the densely packed 10-nm particles observed on the OHC lateral wall are likely to be constituted only of prestin molecules.

Keywords: prestin, GLUT-5, outer hair cell, Chinese hamster ovary cell, transfection, atomic force microscope

\section{INTRODUCTION}

Outer hair cells (OHCs), which are sensory cells in the mammalian cochlea, are able to elongate and contract in length in response to changes in membrane potential (Brownell et al. 1985; Kachar et al. 1986; Ashmore 1987; Santos-Sacchi and Dilger 1988). This motility is believed to amplify the motion of the cochlear partition generated by acoustical stimula- 
tion. As a result, hearing in mammals is characterized by high sensitivity, wide dynamic range, and sharp frequency selectivity. The origin of this motility is associated with a membrane protein in the lateral wall of OHCs (Dallos et al. 1991). The gene that codes this protein was identified from the gerbil cochlea and termed prestin (Zheng et al. 2000). Since its identification, prestin has been researched intensively and has been found to be a direct voltageto-force converter, which uses cytoplasmic anions as extrinsic voltage sensors and can operate at microsecond rates (Dallos and Fakler 2002). The threshold of the auditory brainstem response in prestin knockout mice was revealed to be higher than that of normal mice (Liberman et al. 2002; Cheatham et al. 2004), which indicates that prestin plays an important role in the high sensitivity of hearing.

The lateral wall of the OHC has been observed by electron microscopy (EM). The existence of many particles, $10 \mathrm{~nm}$ in diameter, in the plasma membrane has been shown using the freeze-fracture technique (Arima et al. 1991; Forge 1991; Kalinec et al. 1992; Souter et al. 1995). These densely packed 10-nm particles in the lateral membrane of OHCs are thought to be motor protein. Recently, microstructures of the lateral wall of the OHC have been examined by atomic force microscopy (AFM; Le Grimellec et al. 2002; Wada et al. 2003, 2004). From these AFM observations, the plasma membrane has been confirmed to contain many particles with a diameter of about $10 \mathrm{~nm}$. These particles are likely to be motor protein. In an early study, this motor protein was proposed to be GLUT-5 (Géléoc et al. 1999). Although GLUT-5 has been ruled out as being this motor protein (Belyantseva et al. 2000; Oliver et al. 2001; Liberman et al. 2002), it is unclear whether prestin and GLUT-5 form a complex in the plasma membrane. Therefore, it still remains unknown whether the 10-nm particles seen in EM/AFM images are only prestin or rather a complex of GLUT-5 and prestin molecules.

To determine whether the 10 -nm particles are indeed exclusively constituted of prestin molecules, we examined the plasma membranes of prestin-transfected and untransfected Chinese hamster ovary $(\mathrm{CHO})$ cells, which do not express GLUT-5 (Inukai et al. 1995), using AFM. First, CHO cells on a substrate were shared open by exposure to ultrasonic waves so that only the flat inside-out plasma membranes remained on the substrate. The cytoplasmic faces of the isolated membranes were then observed by AFM in liquid. Analysis of the shape and size of the observed structures was performed in five AFM images of the prestin-transfected $\mathrm{CHO}$ cells and five such images of the untransfected CHO cells. Finally, the difference in the frequency distribution of these structures between the prestin-transfected and untransfected CHO cells was compared.

\section{MATERIALS AND METHODS}

\section{Cells and cell culture}

Prestin-transfected CHO cells, FLAG-tagged prestintransfected $\mathrm{CHO}$ cells, and untransfected $\mathrm{CHO}$ cells were used. The prestin-transfected $\mathrm{CHO}$ cells and FLAG-tagged prestin-transfected $\mathrm{CHO}$ cells were constructed by transfection of gerbil prestin cDNA and such FLAG-tagged prestin cDNA into CHO cells using pIRES-hrGFP-1a mammalian expression vectors (Stratagene, La Jolla, CA, USA), respectively. Those expression vectors contain humanized Renilla reniformis green fluorescent protein (hrGFP) gene, which is connected to the prestin gene. CHO cell lines, which stably express prestin, were established using transfected cells by limiting dilution cloning. The expression and activity of prestin in the generated CHO cell lines were confirmed by immunofluorescence and whole-cell patch-clamp measurements (Iida et al. 2003, 2005). CHO cells, which were not subjected to any transfection procedure, were used as the untransfected $\mathrm{CHO}$ cells.

The prestin-transfected CHO cells, the FLAGtagged prestin-transfected CHO cells, and the untransfected $\mathrm{CHO}$ cells were cultured in RPMI1640 medium with $10 \%$ fetal bovine serum, $100 \mathrm{U}$ penicillin $/ \mathrm{ml}$, and $100 \mu \mathrm{g}$ streptomycin $/ \mathrm{ml}$ at $37^{\circ} \mathrm{C}$ with $5 \% \mathrm{CO}_{2}$. At the beginning of every experimental procedure, the GFP fluorescence of the prestintransfected CHO cells and FLAG-tagged prestintransfected $\mathrm{CHO}$ cells were observed by using a confocal laser scanning microscope (FV500, Olympus, Tokyo, Japan) equipped with a UPlan Apo $20 \times$ $(\mathrm{NA}=0.70)$ objective and an $\operatorname{Ar}$ laser $(488 \mathrm{~nm})$ to confirm the expression of prestin.

\section{Isolation of plasma membranes}

The culture medium was removed, and the cells were detached from a flask by incubation with $100 \mathrm{mM}$ ethylenediaminetetraacetic acid (EDTA)-phosphate buffered saline (PBS) solution. The EDTA-PBS solution containing the cells was put into a tube and centrifuged at $250 \times g$ for $5 \mathrm{~min}$. The supernatant was then removed, and an external solution $(145 \mathrm{mM}$ $\mathrm{NaCl}, 5.8 \mathrm{mM} \mathrm{KCl}, 1.3 \mathrm{mM} \mathrm{CaCl} 2,0.9 \mathrm{mM} \mathrm{MgCl} 2$, $10 \mathrm{mM}$ HEPES, $0.7 \mathrm{mM} \mathrm{Na}_{2} \mathrm{HPO}_{4}$, and $5.6 \mathrm{mM}$ glucose; $\mathrm{pH} 7.3$ ) was put into the tube. The external solution containing the cells was agitated by pipetting in that tube and deposited on glass-bottomed dishes (Asahi Techno Glass, Chiba, Japan) or plastic dishes (Nunc, Roskilde, Denmark) in accordance with the 
intended purpose. After about $10 \mathrm{~min}$, most of the cells had become reattached to the substrate. These cells were sheared open by exposure to ultrasonic waves for a few $100 \mathrm{~ms}$ in a hypotonic buffer $(10 \mathrm{mM}$ PIPES, $10 \mathrm{mM} \mathrm{MgCl}_{2}, 0.5 \mathrm{mM}$ ethyleneglycotetraacetic acid; $\mathrm{pH}$ 7.2) using a sonicator (XL-2000, Misonix, Farmingdale, NY, USA), i.e., sonication. After the sonication, we confirmed that the cell could not be observed by light microscopy but could be observed by staining with a fluorescence lipophilic membrane probe, CM-DiI (Molecular Probes, Eugene, OR, USA), indicating that the cells were indeed sheared open, thus becoming isolated membranes. Furthermore, isolated membranes whose locations remained unchanged after sonication were chosen as samples to ensure that the cytoplasmic faces of the isolated membranes were up. The isolated membranes attached to the substrate were then incubated with a high salt buffer $(2 \mathrm{M} \mathrm{NaCl}, 2.7 \mathrm{mM} \mathrm{KCl}$, $1.5 \mathrm{mM} \mathrm{KH}_{2} \mathrm{PO}_{4}, 1 \mathrm{mM} \mathrm{Na}_{2} \mathrm{HPO}_{4} ; \mathrm{pH} 7.2$ ) for $30 \mathrm{~min}$ at room temperature to remove the cytoskeletal materials and the peripheral proteins (Ziegler et al. 1998). After PBS washing, the isolated membranes were incubated with $0.05 \%$ trypsin for $5 \mathrm{~min}$ at room temperature to remove the remaining materials.

\section{Sample preparation for prestin labeling, lipid} labeling, and low-magnification AFM imaging

In these experiments, the isolated membranes of the FLAG-tagged prestin-transfected $\mathrm{CHO}$ cells and those of the untransfected $\mathrm{CHO}$ cells as described above were used. To prevent autofluorescence of a substrate, glass-bottomed dishes were used. The isolated membranes were fixed with $4 \%$ paraformaldehyde in phosphate buffer for $30 \mathrm{~min}$ at room temperature. After fixation, the membranes were rinsed three times with PBS and incubated with Block Ace (Dainippon Pharmaceutical, Osaka, Japan) for $30 \mathrm{~min}$ at $37^{\circ} \mathrm{C}$. After PBS washing, the membranes were incubated with mouse anti-FLAG primary antibody (Sigma-Aldrich, St. Louis, MO, USA) in PBS for $1 \mathrm{~h}$ at $37^{\circ} \mathrm{C}$. The membranes were then washed with PBS and incubated with fluorescein isothiocyanate (FITC)-conjugated goat anti-mouse IgG secondary antibody (Sigma-Aldrich) in PBS and $2 \mathrm{mM}$ CM-DiI (Molecular Probes), which labels the lipid of the membranes, for $30 \mathrm{~min}$ at $37^{\circ} \mathrm{C}$. Finally, the membranes were washed with PBS and immersed in $0.1 \mathrm{M}$ phosphate buffer solution.

\section{Sample preparation for high-magnification AFM images}

High-magnification AFM images were obtained from the isolated membranes of the prestin-transfected $\mathrm{CHO}$ cells and those of the untransfected CHO cells.
Plastic dishes were used as the substrate. In this case, the isolated membranes were fixed with $1 \%$ glutaraldehyde in PBS for $20 \mathrm{~min}$ at room temperature. The membranes were then incubated with PBS containing $2 \mathrm{mM} \mathrm{CM}$-DiI for $5 \mathrm{~min}$ at $37^{\circ} \mathrm{C}$ and then for $15 \mathrm{~min}$ at $4^{\circ} \mathrm{C}$. Finally, the membranes were washed with PBS and immersed in filtered $0.1 \mathrm{M}$ phosphate buffer solution.

\section{Fluorescence microscopy}

Fluorescence images were obtained from the isolated membranes of the FLAG-tagged prestin-transfected $\mathrm{CHO}$ cells and those of the untransfected $\mathrm{CHO}$ cells by an inverted fluorescence microscope equipped with a Plan Apo $100 \times(\mathrm{NA}=1.40)$ oil-immersion objective and a cooled charge-coupled device camera (DP70, Olympus), which comprise a part of the AFM used in this study. This system enables us to record fluorescence and AFM images of the same sample simultaneously. A mercury lamp was used as a light source. FITC fluorescence was detected with a narrowband filter cube (U-MNIBA, Olympus), which consists of a 470- to 490-nm excitation filter, a 505-nm dichroic mirror, and a 515- to 550-nm emission filter. CM-DiI fluorescence was detected with a wideband filter cube (U-MWIG, Olympus) composed of a 520- to 550-nm excitation filter, a 565-nm dichroic mirror, and a 580-nm emission filter.

\section{Atomic force microscopy}

A commercial AFM (NVB100, Olympus), in which the AFM unit is mounted on an inverted fluorescence microscope (IX70, Olympus), was used. As the AFM unit is mounted on an inverted optical microscope, positioning of the tip above the cells is easy. A $\mathrm{V}$-shaped silicon nitride cantilever (OMCL-TR400PSA2, Olympus) with a spring constant of $0.02 \mathrm{~N} / \mathrm{m}$ was used. The cantilever has a pyramidal tip, the typical radius of its curvature being $16 \pm 1 \mathrm{~nm}$ (personal communication with Olympus). To minimize sample damage during scanning, AFM images were obtained using the oscillation imaging mode (Tapping mode ${ }^{\mathrm{TM}}$, Digital Instruments, Santa Barbara, CA, USA). The cantilever was oscillated near its resonance frequency in liquid $(3.6-4.7 \mathrm{kHz})$. Low-magnification images were obtained from the isolated membranes of the FLAGtagged prestin-transfected $\mathrm{CHO}$ cells and those of the untransfected CHO cells at a scan rate of $0.1 \mathrm{~Hz}$; that is, the scan speeds were 1.0 and $2.0 \mu \mathrm{m} / \mathrm{s}$ when the scan sizes were $5.0 \times 10.0$ and $10.0 \times 10.0 \mu \mathrm{m}$, respectively. High-magnification images (scan size is $1.0 \times 0.5 \mu \mathrm{m}$ ) were obtained from the isolated membranes of the prestin-transfected $\mathrm{CHO}$ cells and those of the untransfected CHO cells. The FLAG-tagged prestin-trans- 
fected CHO cells were not, however, used to take such images because $3 \times$ FLAG peptide (approximately 3 $\mathrm{kDa}$ ) tagged at the $\mathrm{C}$ terminus of prestin is expected to affect the size of prestin when imaging is performed at such high magnification. The scan rate, in this case, was $0.3-0.4 \mathrm{~Hz}$ (scan speed is $0.6-0.8 \mu \mathrm{m} / \mathrm{s}$ ). In all AFM images, the samples were scanned from left to right. Each scan line has 512 points of data, and an image consists of 256 and 512 scan lines in nonsquare and square images, respectively.

\section{Analysis of AFM images}

The original AFM images were flattened by use of a software program (Digital Instruments) to eliminate background slopes and to correct dispersions of individual scanning lines. In this study, differential images were computed from the original flattened AFM images for further analysis of the imaged structures by using Adobe Photoshop 6.0. First, the colored original flattened AFM images were converted into gray-scale AFM images, the intensity value of which ranged from 0 (black) to 255 (white). To remove the noise of the images, spatial averaging was then performed on a $3 \times 3$ pixel neighborhood at each pixel of the images. After averaging, differential operation was performed by a command "find edges." Finally, to more clearly show the structures and minimize background noise in the differential AFM images, contrast of the images was enhanced, resulting in clarification of the edges of the imaged structures so that only the structures with distinct shapes were extracted. Every extracted structure in the calculated differential AFM images was subjected to subsequent analysis of its shape and size, resulting in prevention of experimenter bias.

Atomic force microscopic images are generated by raster scanning with a cantilever across a sample, i.e., left to right and top to bottom in a zigzag pattern. During this scanning, the topological data are continuously obtained in the transversal direction of the AFM image, whereas such data in the longitudinal direction are intermittent. Thus, the topological data in the transversal direction of the image are more reliable than those in the longitudinal direction. The transversal sizes of the observed structures were therefore adopted as their diameters. For analysis of the diameters of the observed structures, a simple geometric model, as shown in Figure 1, was used because such sizes are prone to overestimation due to the radius of the curvature of the AFM tip (Lärmer et al. 1997). When the observed structure was regarded as a sphere in the plasma membrane, the relationship between the diameter of the structure observed by the AFM $(2 \Delta)$ and its actual diameter $(2 r)$ is given by
A

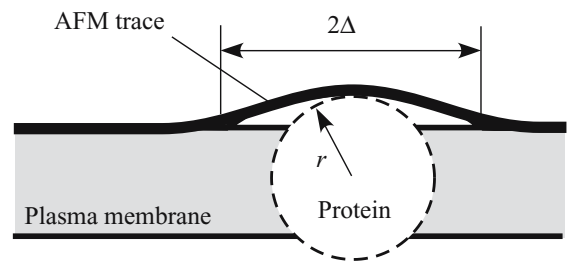

B

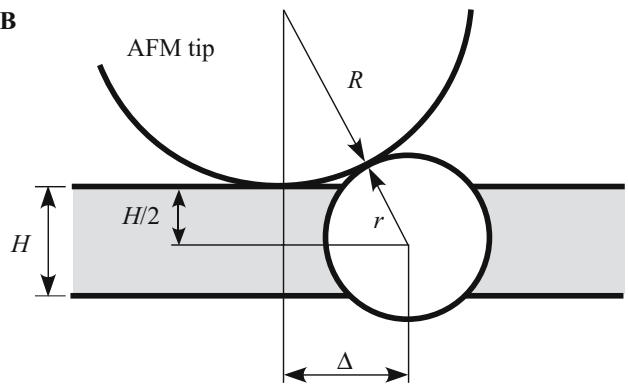

FIG. 1. Sphere model of a membrane protein. (A) AFM trace. The diameter of the protein observed by the AFM $(2 \Delta)$ is larger than the actual diameter (2r). (B) Interaction of the AFM tip with a membrane protein. Using the Pythagorean theorem, the actual diameter $(2 r)$ of the protein can be calculated from the radius $(R)$ of the curvature of the AFM tip, the thickness of the bilayer $(H)$, and the diameter of the protein observed by the AFM (2A).

$$
2 \Delta=2 \sqrt{(R+r)^{2}-(R+H / 2)^{2}}
$$

where $R$ is the radius of the curvature of the AFM tip, which is $16 \pm 1 \mathrm{~nm}$ in the measurements, and $H$ is the thickness of the plasma membrane.

\section{RESULTS}

Expression of prestin in prestin-transfected and FLAG-tagged prestin-transfected $\mathrm{CHO}$ cells

Evaluating the expression of prestin in the transfected CHO cells, the fluorescence of GFP, coexpressed with prestin in such cells, was observed. Figure 2 shows the GFP fluorescence of the prestin-transfected, FLAGtagged prestin-transfected, and untransfected $\mathrm{CHO}$ cells. GFP fluorescence was detected in the prestintransfected (top middle panel) and FLAG-tagged prestin-transfected CHO cells (center panel), indicating the expression of prestin in these cells. However, its intensity varied from cell to cell. By contrast, the GFP fluorescence was not observed in the untransfected CHO cells (bottom middle panel). 


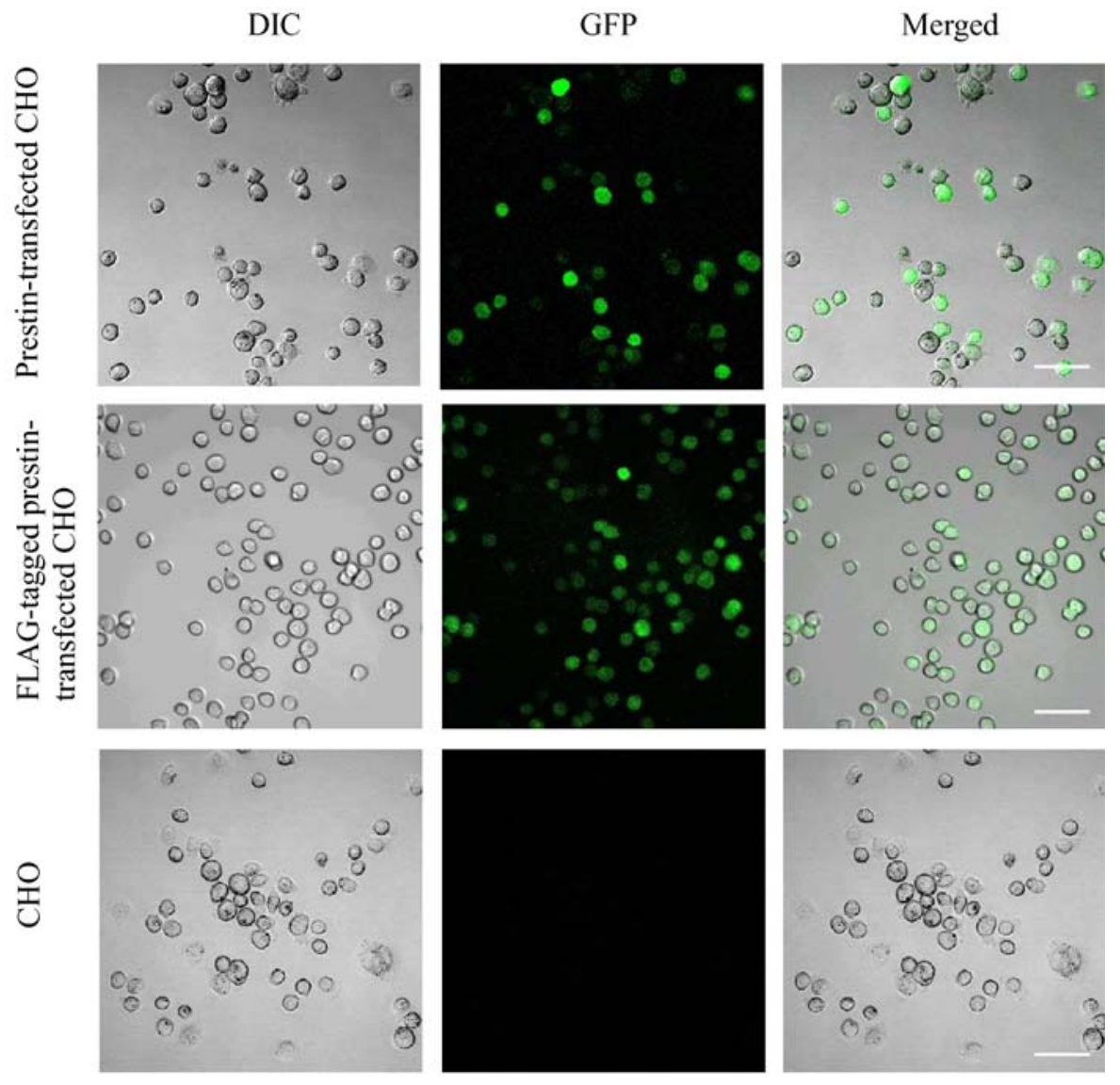

FIG. 2. GFP fluorescence of the prestin-transfected, FLAG-tagged prestin-transfected, and untransfected $\mathrm{CHO}$ cells. Left panels: differential interference contrast (DIC) images; middle panels: GFP fluorescence images; right panels: merged images. Various intensities of GFP fluorescence were observed in the prestin-transfected and

Fluorescence images of prestin and lipid labeling, and low-magnification AFM images of isolated membranes of FLAG-tagged prestin-transfected $\mathrm{CHO}$ cells and those of untransfected $\mathrm{CHO}$ cells

Figure 3 shows the fluorescence images of the prestin and lipid labeling and the corresponding low-magnification AFM images of the isolated membranes of the FLAG-tagged prestin-transfected $\mathrm{CHO}$ cell and those of the untransfected CHO cell. Prestin labeling (green), which labels FLAG peptide tagged at prestin, was observed in the isolated membrane of the FLAG-tagged prestin-transfected CHO cell (Fig. 3A, left panel); by contrast, it was not observed in the isolated membrane of the untransfected $\mathrm{CHO}$ cell (Fig. 3B, left panel). These results indicate the presence of prestin in the isolated membranes of the FLAG-tagged prestin-transfected $\mathrm{CHO}$ cell even if the isolated membranes are sonicated and then incubated with the high salt buffer and trypsin. Lipid labeling (red) is shown in the second panel from the left in both Figure 3A and B. Lipids of both isolated membranes were labeled with CM-DiI. The distribu-
FLAG-tagged prestin-transfected $\mathrm{CHO}$ cell, indicating the variation of the expression level of prestin (top middle and center panels). By contrast, GFP fluorescence was not observed in the untransfected $\mathrm{CHO}$ cells (bottom middle panel). Scale bars are $50 \mu \mathrm{m}$.

tion of CM-DiI in the plasma membrane of the FLAGtagged prestin-transfected $\mathrm{CHO}$ cell was seen as a pattern of bright patches rather than as a uniform pattern. Furthermore, the merged image of the isolated membrane of the FLAG-tagged prestin-transfected CHO cell (Fig. 3A, second panel from the right) revealed that these bright patches of the lipid labeling were colocalized with the prestin labeling, as shown by the yellow region. The magnified image of the boxed area in the merged image is shown at the right. A low-magnification AFM image was obtained from this boxed area (Fig. 3A, right panel). In the AFM image, protruding globular structures, approximately $100 \mathrm{~nm}$ in diameter, were observed. These protruding globular structures corresponded to the colocalized bright patches in the merged image as shown by arrows. This result indicates that prestin is not uniformly distributed in the plasma membrane of the transfected $\mathrm{CHO}$ cells. Rather, a considerable amount of prestin is presumably distributed in some areas of the plasma membrane of such transfected CHO cells. 
A

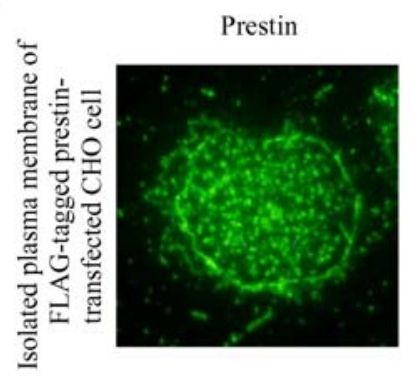

B

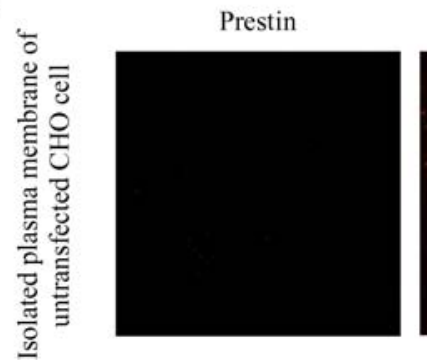

Lipid

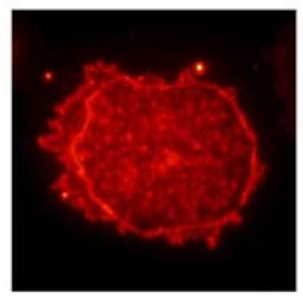

Lipid

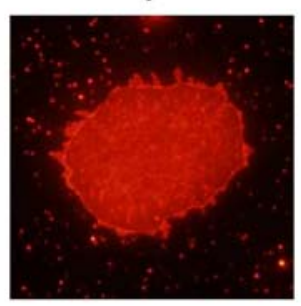

Merged

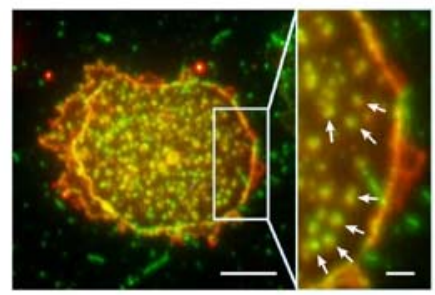

AFM

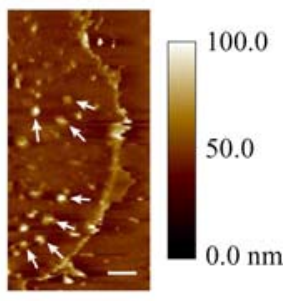

FIG. 3. Fluorescence images of the prestin and lipid labeling, and the corresponding AFM images of the isolated plasma membranes of the $\mathrm{CHO}$ cells. (A) Isolated membrane of a FLAG-tagged prestintransfected $\mathrm{CHO}$ cell. The prestin labeling (left panel, green), which was visualized by incubation with mouse anti-FLAG primary antibody and FITC-conjugated goat anti-mouse IgG secondary antibody, was colocalized with the lipid labeling (second panel from the left, red), which was visualized by incubation with CM-Dil, as shown by the yellow region in the merged image (second panel from the right). A magnified image of the boxed area in the merged image is shown to the right. A low-magnification AFM image was

\section{High-magnification AFM images}

Substrate and isolated plasma membranes of $\mathrm{CHO}$ cells. To obtain high-magnification AFM images of the CHO plasma membranes, plastic dishes were used as substrate when the AFM observation was carried out. To evaluate the validity of plastic dishes as substrate, the topography of the surface of such dishes was examined at high-magnification imaging. Figure 4A shows the AFM image of the surface of the plastic dish, and Figure $4 \mathrm{~B}$ shows the section along the dotted line denoted in Figure 4A. Mean roughness of the surface of the plastic dish was less than $0.5 \mathrm{~nm}$ over the scan length of $1.0 \mu \mathrm{m}$. Figure $5 \mathrm{~A}$ shows the AFM image of the edge of the isolated plasma membrane of the untransfected $\mathrm{CHO}$ cell after incubation with the high salt buffer and trypsin. Figure $5 \mathrm{~B}$ indicates the cross section along the dotted line shown in Figure 5A. From the cross section, although a large structure exists at the edge of the membrane (arrow), the average thickness of the plasma membrane was estimated to be approximately $5 \mathrm{~nm}$.

Effects of high salt buffer and trypsin on isolated plasma membranes of $\mathrm{CHO}$ cells. In this study, the plasma membranes of the cells were isolated on the substrate

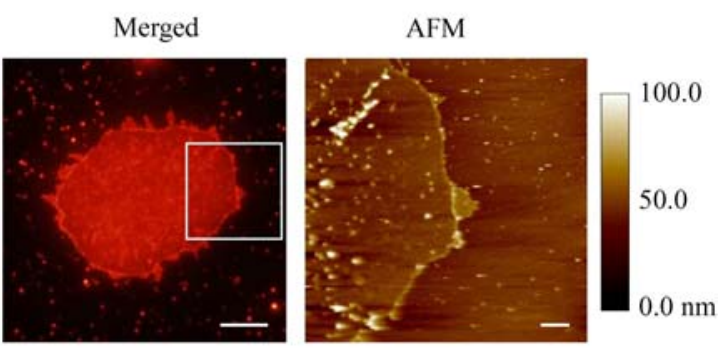

obtained from the boxed area. Globular structures approximately $100 \mathrm{~nm}$ in diameter were observed. They correspond to the colocalized bright patches in the merged image as indicated by the arrows. (B) Isolated plasma membrane of an untransfected $\mathrm{CHO}$ cell. The prestin labeling was not detected (left panel). The lipid labeling was observed (second panel from the left, red). A lowmagnification AFM image was obtained from the boxed area indicated in the merged image. Scale bars of the fluorescence images, the magnified image in A, and the AFM images are 5.0, 1.0, and $1.0 \mu \mathrm{m}$, respectively.

by sonication. Although it was essential for this study to obtain plasma membrane containing only prestin, cytoskeletal materials and peripheral proteins may remain on the cytoplasmic face of the plasma membrane by this preparation. To remove such materials, incubation with the high salt buffer was performed. To confirm the effects of such incubation on the isolated plasma membrane, AFM images of the plasma membranes of the untransfected $\mathrm{CHO}$ cells were obtained after the incubation. Figure $6 \mathrm{~A}$ represents the AFM image of the isolated plasma membrane after incubation with the high salt buffer. Large structures approximately $100 \mathrm{~nm}$ in diameter (arrows) were observed in this image. Because these large structures were thought to be residual cytoskeletal materials and/ or peripheral proteins, the membranes were then additionally incubated with $0.05 \%$ trypsin. As a result, such large structures were hardly seen as shown in Figure 6B.

Membrane proteins in isolated plasma membranes of prestin-transfected $\mathrm{CHO}$ cells and those of untransfected CHO cells. The cytoplasmic surfaces of the isolated plasma membranes of the prestin-transfected $\mathrm{CHO}$ cells and those of the untransfected CHO cells were observed by the tapping mode of AFM to visualize the 
A

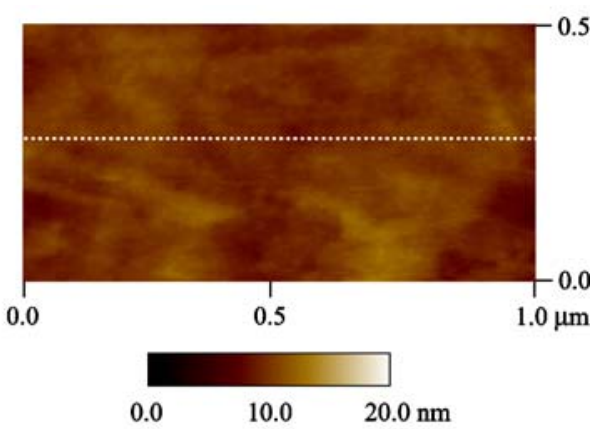

B

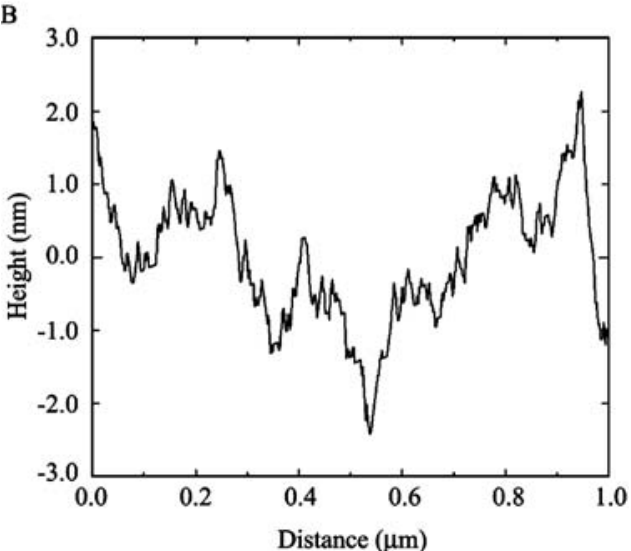

FIG. 4. Topography of the surface of the plastic dish. (A) The AFM image. (B) The section along the dotted line shown in A. Mean roughness of the surface is less than $0.5 \mathrm{~nm}$ over the scan length of $1.0 \mu \mathrm{m}$.

membrane proteins. Figure 7 represents their original flattened AFM images and the calculated differential AFM images. As indicated by arrows, particle-like structures were recognized in the plasma membranes of both cells; however, no distinctive difference in such particle-like structures was found between the prestintransfected $\mathrm{CHO}$ cells and the untransfected $\mathrm{CHO}$ cells. Analysis of the shape and size of the observed structures was then performed in five AFM images of the prestin-transfected $\mathrm{CHO}$ cells and five such images of the untransfected CHO cells. The existence of particle-like structures was detected by local peaks in the original flattened AFM images (Fig. 7A1 and B1), and the size of these structures was determined from the differential AFM images (Fig. 7A2 and B2). In this study, when the size of the particle-like structures was over $100 \mathrm{~nm}$, they were neglected because the size of the membrane proteins is on the order of tens of nanometers. When the shape index, i.e., the ratio of the minor axis length to the major axis length, was $0.5-1.0$, the structure was regarded as being a particle-like structure. Arrowheads indicate structures that did not meet such criteria. In addition, a simple geometric model (Fig. 1) was used for the analysis of the transversal size of the observed structures because they are prone to overestimation due to the radius of the curvature of the AFM tip; thus, such sizes were compensated by Eq. (1). The radius of the curvature of the AFM tip $R$ was $16 \pm 1 \mathrm{~nm}$. Assuming that the radius of the curvature of the cantilever tip changed by $1.0 \mathrm{~nm}$, the change in the diameter of the particle-like structure is $1.0 \mathrm{~nm}$ based on the geometric model proposed in the present study. Such changes in the diameter are less than the resolution of the AFM in this experiment. Therefore, the effect of the dispersion of the radius of the curvature is negligibly small. Thus, in the present study, the radius of the curvature of the AFM tip $R$ was assumed to be $16 \mathrm{~nm}$. Regarding the thickness of the plasma membrane, it is known that expression of membrane proteins affects the thickness of the plasma membrane, which is on the order of

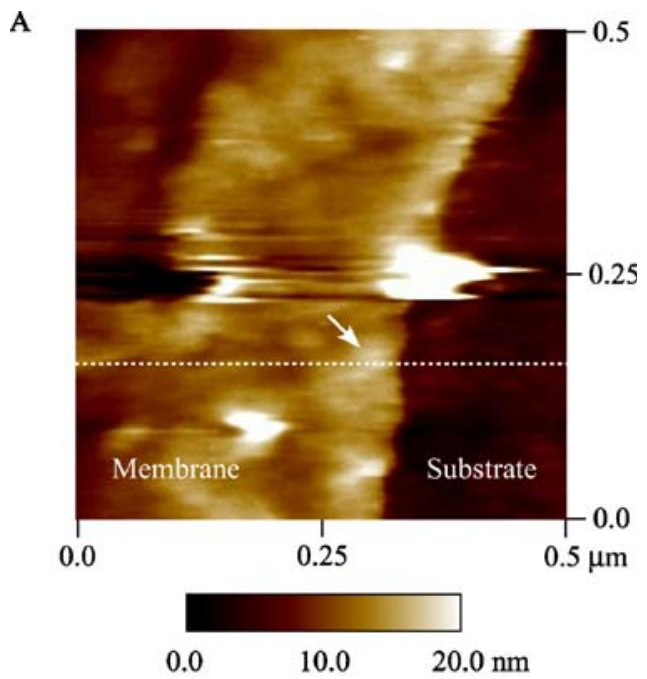

B

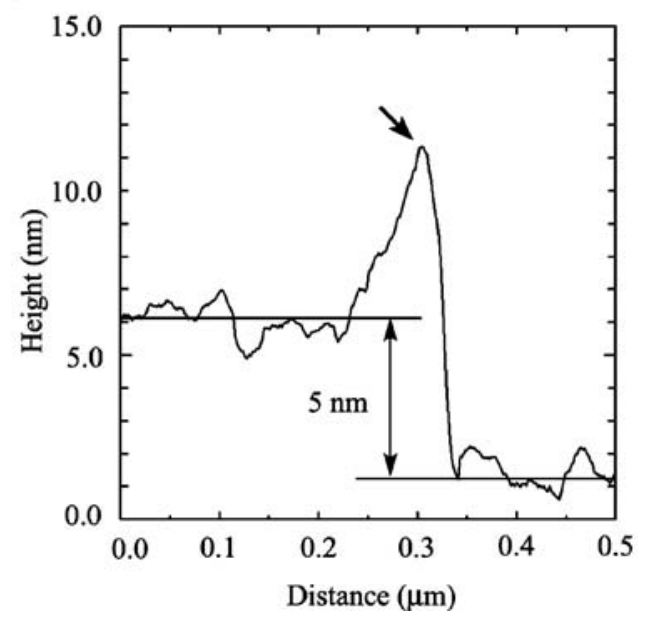

FIG. 5. Thickness of the plasma membrane of the untransfected $\mathrm{CHO}$ cell after incubation with the high salt buffer and trypsin. (A) AFM image of the edge of the isolated membrane. (B) Cross section along the dotted line shown in A. Although a large structure was observed at the edge of the membrane (arrow), the averaged thickness of the membrane was estimated to be approximately $5 \mathrm{~nm}$. 

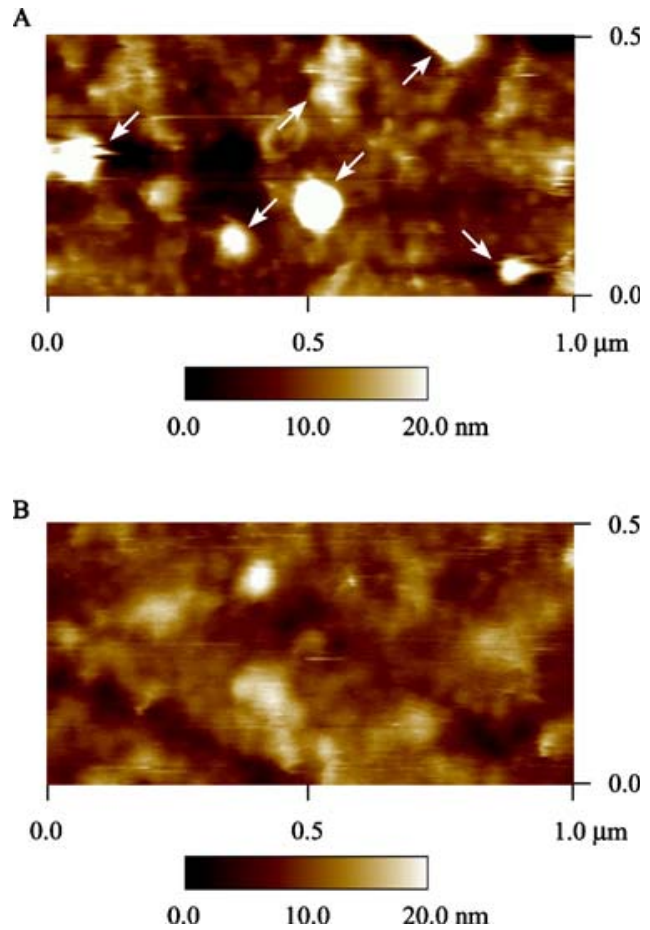

FIG. 6. Effect of the high salt buffer and trypsin on the isolated membrane of the untransfected $\mathrm{CHO}$ cell. (A) AFM image of the membrane after incubation with the high salt buffer. Arrows indicate large structures approximately $100 \mathrm{~nm}$ in diameter, thought to be residual cytoskeletal material and/or peripheral protein. (B) AFM image of the membrane after incubation with the high salt buffer and $0.05 \%$ trypsin. Large structures (arrows) seen in A are hardly seen in B.

angstroms (Mitra et al. 2004). Assuming that the thickness of the plasma membrane increased by $1.0 \mathrm{~nm}$ because of the expression of prestin, the increase in the diameter of the particle-like structure is $1.0 \mathrm{~nm}$ based on the proposed geometric model. Such increase is less than the resolution of the AFM used in this experiment. Therefore, in the present study, because the effect of membrane proteins on the thickness of the plasma membrane is negligibly small, the thickness of the plasma membrane $H$ was determined to be $5 \mathrm{~nm}$ (data from Fig. 5). The frequency distribution of the observed particle-like structures, i.e., the density of the particle-like structures plotted against the interval of 2-nm classes in the diameter, is shown in Figure 8. The diameters of the particlelike structures of the prestin-transfected $\mathrm{CHO}$ cells ranged from 6 to $40 \mathrm{~nm}$, and those of the untransfected CHO cells ranged from 6 to $30 \mathrm{~nm}$. When the sizes of the particle-like structures in the plasma membranes were 8-10 and 10-12 $\mathrm{nm}$, the differences of their densities between the prestin-transfected $\mathrm{CHO}$ cells and the untransfected $\mathrm{CHO}$ cells were statistically significant for $P<0.05$ using the MannWhitney test, as indicated by the asterisks.

\section{DISCUSSION}

Membrane preparation process

In this study, to obtain isolated plasma membranes, the cells were sheared open by sonication, and the cytoplasmic surfaces were exposed. However, cytoskeletal materials and peripheral proteins may remain on the cytoplasmic surface with such treatment (Shurety et al. 1998; Avery et al. 2000; Wetzel et al. 2003). Such residue is thought to obstruct observation of the membrane proteins. To remove these materials, incubation of isolated plasma membranes with the high salt buffer was performed because such incubation leads to the disruption of the ionic and hydrogen bonds of the proteins (Ziegler et al. 1998), which contribute to their three-dimensional structure such as the $\alpha$ helix, $\beta$ sheet, etc. Thus, cytoskeletal materials and peripheral proteins could be removed by incubation with the high salt buffer. In this study, however, when the isolated membrane was observed by AFM after incubation with the high salt buffer, large structures were seen (Fig. 6A). Because these large structures were thought to be the remains of the cytoskeletal material and/or the peripheral proteins, the membranes were additionally incubated with trypsin, as a kind of protease, and then observed by AFM. As a result, the large structures were hardly seen (Fig. 6B), suggesting that the incubation with trypsin seems to be effective in removing the residual cytoskeletal materials and/or membrane proteins.

However, the effect of trypsin on prestin must be taken into account. Kalinec et al. (1992) reported that the cytoplasmic structure was disrupted by the injection of trypsin into the OHC; however, the electromotility of the OHC, indicating the activity of prestin, was not diminished. Moreover, it has recently been reported that the voltage-dependent nonlinear capacitance, which is thought to indicate the motor activity of prestin, was measured from prestin-transfected human embryonic kidney cells despite trypsin being injected into the cells (Dong and Iwasa 2004). These reports suggest that the structure of prestin is not degraded and deformed by trypsin. Indeed, the presence of prestin in the isolated plasma membranes of the FLAG-tagged prestin-transfected CHO cells was confirmed by an indirect immunofluorescence experiment after isolated membranes were sonicated and incubated with the high salt buffer and $0.05 \%$ trypsin, as shown in the left panel of Figure 3A. These results are thought to be much the same for the prestin-transfected CHO cells.

\section{Imaging of membrane protein prestin}

In attempting to visualize prestin by AFM, the expression level of prestin in the prestin-transfected CHO 
Al

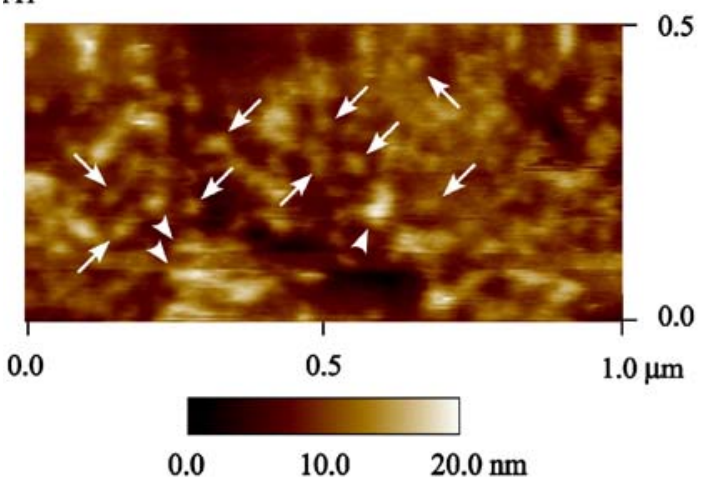

A2

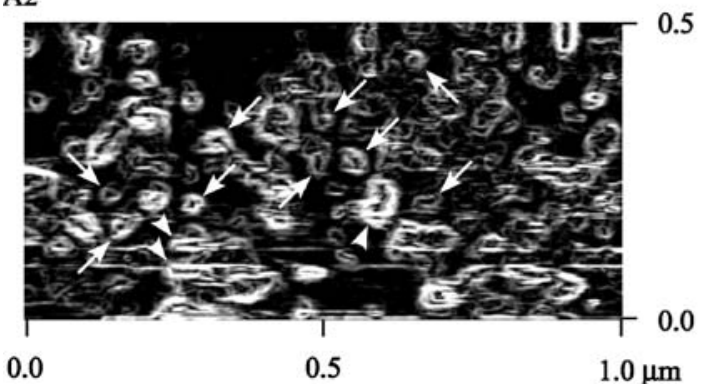

FIG. 7. AFM images of membranes of the $\mathrm{CHO}$ cells. (A1) Original flattened AFM image of the prestin-transfected $\mathrm{CHO}$ cell. (A2) Differential AFM image of A1. (B1) Original flattened AFM image of the untransfected $\mathrm{CHO}$ cell. (B2) Differential AFM image of B1. Arrows indicate particle-like structures recognized in the plasma

cells should be taken into account. Iida et al. (2004) reported that there is a positive correlation between the intensity of the GFP fluorescence and the expression level of prestin in the prestin-transfected $\mathrm{CHO}$ cells. GFP fluorescence was therefore first examined as an index of the expression level of prestin before the AFM observation (Fig. 2, top middle panel), and the cells with the high intensity of the GFP fluorescence were then observed by AFM.

As shown by the arrows in the right panel of Figure 3A, the low-magnification images demonstrated that protruding globular structures approximately $100 \mathrm{~nm}$ in diameter were distributed over the plasma membranes of the FLAG-tagged prestin-transfected $\mathrm{CHO}$ cells and the untransfected $\mathrm{CHO}$ cells. Such protruding structures can possibly greatly hinder efforts to obtain AFM images at high magnification because the rapid change in the topography results in reduction of the image resolution near its peripheral areas. The images were therefore obtained in the membrane areas without the protruding structures; that is, when such 100-nm protruding structures were recognized during imaging, the scanning position was adjusted to different nearby areas.

The topography of the surface of the substrate should also be considered because the AFM image
B1

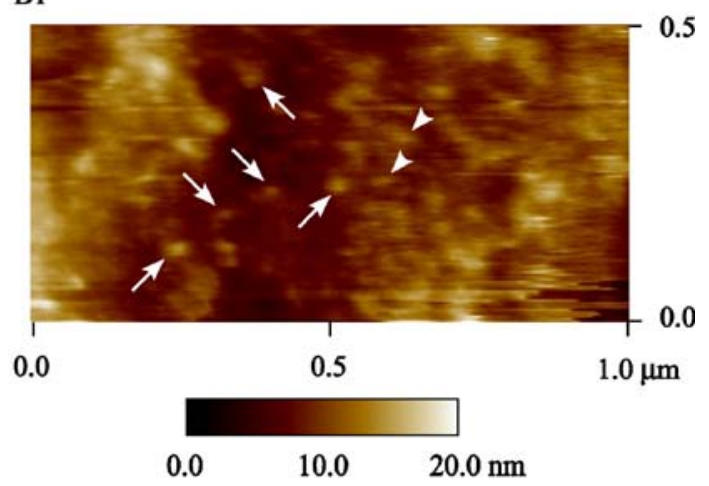

B2

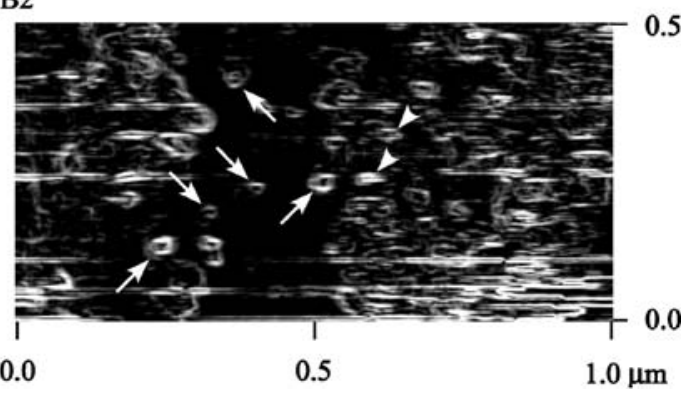

membranes of the prestin-transfected $\mathrm{CHO}$ cells and the untransfected $\mathrm{CHO}$ cells, whereas arrowheads indicate structures that did not meet the criteria for particle-like structures. However, no distinctive difference in such particle-like structures was found between these cells.

shows not only the topography of the surface of the membrane but also that of the substrate, especially in the case of high-magnification imaging. In this study, plastic dishes were used as the substrate for high-magnification imaging. As shown in Figure 4, the mean roughness of the surface of the plastic dish was less than $0.5 \mathrm{~nm}$ over the scan length of $1.0 \mu \mathrm{m}$. Because the size of the membrane proteins is thought to be several tens of nanometers, the effect of the surface of the plastic dishes on the AFM image of the membrane is negligible. The plastic dish was therefore usable as the substrate.

To evaluate the validity of the method of obtaining the plasma membrane, section analysis of the prepared samples was carried out. This analysis of the samples elucidated that the isolated plasma membranes left on the substrate were approximately $5 \mathrm{~nm}$ thick, being consistent with their generally accepted thickness, i.e., 3-10 nm, whereas some large heterogeneous structures remained at the edge of the isolated membrane (Fig. 5). These heterogeneous structures that accumulated at the edge of the isolated membrane were associated with the brightly stained part along the edge of the plasma membrane with CM-DiI (Fig. 3A and B, second panels from the left), indicating the place where the plasma mem- 
branes are abundantly aggregated (Thomas et al. 1994). Such aggregation of the plasma membranes at their edge is presumably caused by the application of sonication.

Particle-like structures in high-magnification AFM images of the prestin-transfected $\mathrm{CHO}$ cells and those of the untransfected $\mathrm{CHO}$ cells

In this study, particle-like structures were recognized both in the AFM images of the isolated plasma membranes of the prestin-transfected $\mathrm{CHO}$ cells and in those of the untransfected CHO cells (Fig. 7). It has been reported that randomly distributed intramembrane particles, $10 \mathrm{~nm}$ in size, were seen in the electron microscopic image of the plasma membrane P-fracture face from CHO cells (Yang et al. 1996; Van Hoek et al. 1998). Particles have also been observed in the AFM images of the isolated cytoplasmic plasma membranes of many kinds of cells (Ziegler et al. 1998; Ehrenhöfer et al. 1997; Le Grimellec et al. 1995; Schillers et al. 2001). These particles are believed to be membrane proteins. The particle-like structures in the plasma membranes of the prestin-transfected $\mathrm{CHO}$ cells and the untransfected $\mathrm{CHO}$ cells observed in this study were therefore considered to be such membrane proteins.

However, because there are many kinds of membrane proteins in the plasma membrane of the $\mathrm{CHO}$ cells, it is impossible to clarify whether the observed structures are prestin or not. Analysis of the shape and size of the observed structures was therefore performed for both AFM images of the prestin-transfected $\mathrm{CHO}$ cells and those of the untransfected $\mathrm{CHO}$ cells. As a result, the densities of the particle-like structures in the plasma membrane were found to differ between the prestintransfected $\mathrm{CHO}$ cells and the untransfected $\mathrm{CHO}$ cells; that is, statistical analysis indicated a significant difference in the density of the particle-like structures with diameters of $8-10$ and $10-12 \mathrm{~nm}$ between the prestintransfected $\mathrm{CHO}$ cells and the untransfected $\mathrm{CHO}$ cells for $P<0.05$ using the Mann-Whitney test, as shown by the asterisks in Figure 8. Because the difference between the prestin-transfected and untransfected $\mathrm{CHO}$ cells is caused by the existence of prestin, the difference of the densities of the particle-like structures between the prestin-transfected $\mathrm{CHO}$ cells and the untransfected $\mathrm{CHO}$ cells is considered to be caused by the presence or absence of prestin. Based on Figure 8, therefore, the density of prestin in the prestin-transfected CHO cells was estimated to be $18 \pm 9$ proteins $/ \mu \mathrm{m}^{2}(n=5)$ after subtracting the value of the density of the particlelike structures in the untransfected $\mathrm{CHO}$ cells from those in the prestin-transfected $\mathrm{CHO}$ cells in the 8- to $12-n m$ class. This value corresponds to approximately $75 \%$ of the total density of the particle-like structures

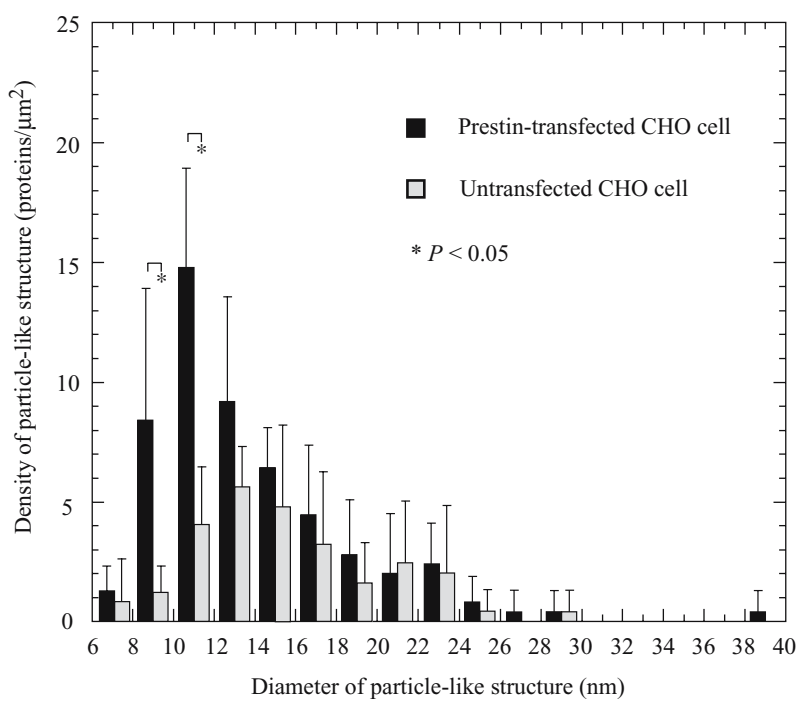

FIG. 8. Frequency distribution of the observed particle-like structures in the plasma membrane. The density of the particle-like structures is plotted against the interval in 2-nm classes. Data were obtained from five AFM images of the prestin-transfected $\mathrm{CHO}$ cells and five such images of the untransfected $\mathrm{CHO}$ cells. When the sizes of the particle-like structures were 8-10 and 10-12 nm, differences of their densities between the prestin-transfected $\mathrm{CHO}$ cells and the untransfected $\mathrm{CHO}$ cells were statistically significant for $P<0.05$ using the Mann-Whitney test, as shown by the asterisks. Error bars represent standard deviations.

in the prestin-transfected $\mathrm{CHO}$ cell membrane. These results suggest that the majority of these particle-like structures with a diameter of 8-12 $\mathrm{nm}$ in the prestintransfected $\mathrm{CHO}$ plasma membrane are possibly prestin.

Previous morphological studies of OHCs using EM and AFM revealed the lateral membrane of OHCs to be densely covered with particles approximately $10 \mathrm{~nm}$ in diameter (Arima et al. 1991; Forge 1991; Kalinec et al. 1992: Souter et al. 1995; Le Grimellec et al. 2002), these particles being believed to be motor protein. Two membrane proteins, i.e., a fructose transporter, GLUT-5 (Géléoc et al. 1999), and an anion/ sulfate transporter, prestin (Zheng et al. 2000), were proposed to be this motor. Although recent studies have ascertained that the motor protein is prestin, not GLUT-5 (Belyantseva et al. 2000; Oliver et al. 2001; Liberman et al. 2002), constituents of the 10-nm particles observed in the $\mathrm{OHC}$ lateral membrane have remained unclear; in other words, it is unknown whether these 10-nm particles are indeed exclusively constituted of prestin molecules or rather a complex of GLUT- 5 and prestin molecules. In the present study, we found particle-like structures with a diameter of $8-12 \mathrm{~nm}$ in the prestin-transfected CHO plasma membrane. As CHO cells do not express GLUT-5 (Inukai et al. 1995), the 8- to 12-nm particle-like structures observed in this study are constituted only of prestin. These diameters are identical to those of the 
high-density particles $(\sim 10 \mathrm{~nm})$ observed in the lateral membrane of the OHC by EM/AFM. These results imply that the densely packed 10 -nm particles in the lateral membrane of OHCs are exclusively composed of prestin molecules, as is the case of the particle-like structures of the prestin-transfected CHO cells.

In addition, in the present study, we imaged the surface topology of the cytoplasmic face of the plasma membrane of the prestin-transfected CHO cells. Because the exact tertiary structure of prestin has not yet been clarified, it is difficult to specify which structure of prestin on the cytoplasmic face was imaged. However, discussion of this issue is thought to provide some useful information on a possible prestin structure. On the cytoplasmic face of the plasma membrane of the prestin-transfected CHO cells, two large fragments protrude, i.e., N- (about 100 amino acids) and C-termini (about 250 amino acids; Zheng et al. 2001). Among these termini, the STAS (after sulfate transporter and antisigma factor antagonist) domain (amino acids 525-713), located at the C-terminus of prestin (Dallos and Fakler 2002), possibly occupies a large conformational space of the cytoplasmic region of prestin because its estimated size based on its molecular weight (about $21 \mathrm{kDa}$ ) is approximately $4 \mathrm{~nm}$ in diameter if its shape is assumed to be cubical (Edstrom et al. 1990). The imaged particle-like structures are therefore thought to predominantly reflect the shape of the STAS domain rather than that of whole molecule of prestin. Assuming that prestin comprises a homo-oligomeric complex in the plasma membrane, these structural presumptions and our experimental data indicate that prestin may possibly form such an oligomer with about two to five prestin monomers based on a geometrical relationship.

Difference between the estimated density of prestin in the prestin-transfected $\mathrm{CHO}$ cells by AFM and that by electrophysiological measurement

According to this study, the density of prestin in the prestin-transfected $\mathrm{CHO}$ cells was obtained to be $18 \pm$ 9 proteins $/ \mu^{2}(n=5)$ as mentioned above. However, the estimated density of prestin in the prestintransfected $\mathrm{CHO}$ cells was $246 \pm 125$ proteins $/ \mu \mathrm{m}^{2}$ ( $n=$ 20) by electrophysiological measurement (Iida et al. 2005). The difference between the density obtained by the AFM and that estimated by electrophysiological measurement may be caused by the fact that the membrane proteins observed by the AFM tend to be undercounted because these proteins comprise complexes (Ehrenhöfer et al. 1997; Schillers et al. 2001) and/or are close to each other. Another possible reason is that membrane proteins approximately $10 \mathrm{~nm}$ in size, including prestin, were rarely distributed in the areas that were scanned at a high magnification by AFM in this study, i.e., the areas without 100-nm protruding globular structures. Thomas et al. (1994) reported that there is correspondence between the lipid labeling with the lipophilic membrane probes, such as CM-DiI and octadecyl rhodamine $\mathrm{B}$, and the membrane part with the abundant membrane proteins. This coaggregation of lipid probes that accompanies the aggregation of membrane proteins reflects a change in plasma membrane structure, where the assembly and/or disassembly of the vesicles might be occurred. Interestingly, as shown in Figure 3A, the globular structures with a diameter of approximately $100 \mathrm{~nm}$ observed by AFM also corresponded to the colocalized bright patches of the prestin and lipid labeling as shown by arrows. These results suggest that these protrusive $100-\mathrm{nm}$ patches possibly correspond to vesicles in progress such as the clathrincoated pits (Van Hoek et al. 1998; Le Grimellec et al. 1995), leading to the aggregation of prestin and other membrane proteins in such pits. Thus, in this study, the density of prestin was presumed to be underestimated.

\section{CONCLUSIONS}

Membranes of prestin-transfected and untransfected CHO cells, which do not express GLUT-5, were observed by AFM. More particle-like structures with a diameter of 8-12 nm exist in the plasma membrane of the prestin-transfected $\mathrm{CHO}$ cells than in that of the untransfected CHO cells. Approximately $75 \%$ of such particle-like structures with a diameter of 8-12 $\mathrm{nm}$ are possibly formed only by prestin molecules, suggesting that the densely packed $10-\mathrm{nm}$ particles in the lateral membrane of OHCs are exclusively constituted of prestin.

\section{ACKNOWLEDGMENTS}

The authors are grateful to Professor M. Sokabe of the Department of Physiology at Nagoya University Graduate School of Medicine in Japan for valuable advice about the membrane preparations, Dr. D. N. Furness of Mackay Institute of Communication and Neuroscience at Keele University in UK for valuable advice about analysis of the AFM images, Mr. A. Yagi of Olympus Co. in Japan for the technical assistance, and Dr. D. Z. Z. He of the Department of Biomedical Sciences at Creighton University in USA for helpful suggestions and comments on the manuscript. This work was supported by Grant-in-Aid for Scientific Research on Priority Areas 15086202 from the Ministry of Education, Culture, Sports, Science and Technology of Japan, by a Health and Labour Science Research Grant from the Ministry of Health, Labour and Welfare of Japan, by a grant from the Human Frontier Science Program to H.W., and by the Special Research Grant 11170012 from the Tohoku University 21st 
Century COE Program of the "Future Medical Engineering Based on Bio-nanotechnology" to M.M.

\section{REFERENCES}

Arima T, Kuraoka A, Toriya R, Shibata Y, Uemura T. Quick-freeze, deep-etch visualization of the 'cytoskeletal spring' of cochlear outer hair cells. Cell Tissue Res. 263:91-97, 1991.

AsHmoRe JF. A fast motile response in guinea-pig outer hair cells: the cellular basis of the cochlear amplifier. J. Physiol. 388:323$347,1987$.

Avery J, Ellis DJ, Lang T, Holroyd P, Riedel D, Henderson RM, EDWARDSON JM, JAHN R. A cell-free system for regulated exocytosis in PC12 cells. J. Cell Biol. 148:317-324, 2000.

Belyantseva IA, Adler HJ, Curi R, Frolenkov GI, Kachar B. Expression and localization of prestin and the sugar transporter GLUT-5 during development of electromotility in cochlear outer hair cells. J. Neurosci. 20:RC116, 2000.

Brownell WE, Bader CR, Bertrand D, De Ribaupierre Y. Evoked mechanical responses of isolated cochlear outer hair cells. Science 227:194-196, 1985.

Cheatham MA, Huynh KH, Gao J, Zou J, Dallos P. Cochlear function in prestin knockout mice. J. Physiol. 560:821-830, 2004.

Dallos P, Fakler B. Prestin, a new type of motor protein. Nat. Mol. Cell Biol. 3:104-111, 2002.

Dallos P, Evans BN, Hallworth R. Nature of the motor element in electrokinetic shape changes of cochlear outer hair cells. Nature 350:155-157, 1991.

DONG XX, IwASA KH. Tension sensitivity of prestin: comparison with membrane motor in outer hair cells. Biophys. J. 86:1201-1208, 2004.

Edstrom RD, Meinke MH, Yang X, Yang R, Elings V, Evans DF. Direct visualization of phosphorylase-phosphorylase kinase complexes by scanning tunneling and atomic force microscopy. Biophys. J. 58:1437-1448, 1990.

Ehrenhöfer U, Rakowska A, Schneider SW, Schwab A, Oberleithner H. The atomic force microscope detects ATP-sensitive protein clusters in the plasma membrane of transformed MDCK cells. Cell Biol. Int. 21:737-746, 1997.

Forge A. Structural features of the lateral walls in mammalian cochlear outer hair cells. Cell Tissue Res. 265:473-483, 1991.

Géléoc GSG, Casalotti SO, Forge A, Ashmore JF. A sugar transporter as a candidate for the outer hair cell motor. Nat. Neurosci. 2:713-719, 1999.

Iida K, Konno K, Oshima T, Tsumoto K, Ikeda K, Kumagai I, Kobayashi T, WADA H. Stable expression of the motor protein prestin in Chinese hamster ovary cells. JSME Int. J. 46C:1266$1274,2003$.

Iida K, Nagaoka T, Tsumoto K, Ikeda K, Kumagai I, Kobayashi T, WADA H. Relationship between fluorescence intensity of GFP and the expression level of prestin in a prestin-expressing Chinese hamster ovary cell line. JSME Int. J. 47C:970-976, 2004.

Iida K, Tsumoto K, Ikeda K, Kumagai I, Kobayashi T, Wada H. Construction of an expression system for the motor protein prestin in Chinese hamster ovary cells. Hear. Res. 205:262-270, 2005.

Inukai K, Katagiri H, Takata K, Asano T, Anai M, Ishihara H, NAKAZAKI M, KikUCHI M, YAZAKI Y, OKa Y. Characterization of rat GLUT-5 and functional analysis of chimeric proteins of GLUT-1 glucose transporter and GLUT-5 fructose transporter. Endocrinology 136:4850-4857, 1995.

Kachar B, Brownell WE, Altschuler R, Fex J. Electrokinetic shape changes of cochlear outer hair cells. Nature 322:365-368, 1986.

Kalinec F, Holley MC, Iwasa KH, Lim DJ, Kachar B. A membranebased force generation mechanism in auditory sensory cells. Proc. Natl. Acad. Sci. USA 89:8671-8675, 1992.
Lärmer J, Schneider SW, Danker T, Schwab A, Oberleithner H. Imaging excised apical plasma membrane patches of MDCK cells in physiological conditions with atomic force microscopy. Pflügers Arch. 434:254-260, 1997.

Le Grimellec C, Lesniewska E, Giocondi MC, Cachia C, Schreiber JP, Goudonnet JP. Imaging of the cytoplasmic leaflet of the plasma membrane by atomic force microscopy. Scanning Microsc. 9:401-411, 1995.

Le Grimellec C, Giocondi MC, Lenoir M, Vater M, Sposito G, Pujol R. High-resolution three-dimensional imaging of the lateral plasma membrane of cochlear outer hair cells by atomic force microscopy. J. Comp. Neurol. 451:62-69, 2002.

Liberman MC, Gao J, He DZZ, Wu X, Jia S, Zuo J. Prestin is required for electromotility of the outer hair cell and for the cochlear amplifier. Nature 419:300-304, 2002.

Mitra K, Ubarretxena-Belandia I, Taguchi T, Warren G, Engelman DM. Modulation of the bilayer thickness of exocytic pathway membranes by membrane proteins rather than cholesterol. Proc. Natl. Acad. Sci. USA 101:4083-4088, 2004.

Oliver D, He DZZ, Klöcker N, Ludwig J, Schulte U, Waldegger S, Ruppersberg JP, Dallos P, FAKLer B. Intracellular anions as the voltage sensor of prestin, the outer hair cell motor protein. Science 292:2340-2343, 2001.

Santos-Sacchi J, Dilger JP. Whole cell currents and mechanical responses of isolated outer hair cells. Hear. Res. 35:143-150, 1988.

Schillers H, Danker T, Madeja M, Oberleithner H. Plasma membrane protein clusters appear in CFTR-expressing Xenopus Laevis Oocytes after cAMP stimulation. J. Membr. Biol. 180:205-212, 2001.

Shurety W, Stewart NL, STOw JL. Fluid-phase markers in the basolateral endocytic pathway accumulate in response to the actin assembly-promoting drug Jasplakinolide. Mol. Biol. Cell 9:957975, 1998.

Souter M, Nevill G, Forge A. Postnatal development of membrane specialisations of gerbil outer hair cells. Hear. Res. 91:43-62, 1995.

Thomas JL, Holowka D, Baird B, WebB WW. Large-scale co-aggregation of fluorescent lipid probes with cell surface proteins. J. Cell Biol. 125:795-802, 1994.

VAN Hokk AN, YANG B, KIRMiz S, Brown D. Freeze-fracture analysis of plasma membranes of $\mathrm{CHO}$ cells stably expressing aquaporins 1-5. J. Membr. Biol. 165:243-254, 1998.

Wada H, Usukura H, Sugawara M, Katori Y, Kakehata S, Ikeda K, Kobayashi T. Relationship between the local stiffness of the outer hair cell along the cell axis and its ultrastructure observed by atomic force microscopy. Hear. Res. 177:61-70, 2003.

Wada H, Kimura K, Gomi T, Sugawara M, Katori Y, Kakehata S, Ikeda $\mathrm{K}$, KobaYASH $\mathrm{T}$. Imaging of the cortical cytoskeleton of guinea pig outer hair cells using atomic force microscopy. Hear. Res. 187:52, 2004.

Wetzel DM, Håkansson S, Hu K, Roos D, Sibley LD. Actin filament polymerization regulates gliding motility by apicomplexan parasites. Mol. Biol. Cell 14:396-406, 2003.

YAng B, Brown D, Verkman AS. The mercurial insensitive water channel (AQP-4) forms orthogonal arrays in stably transfected Chinese hamster ovary cells. J. Biol. Chem. 271:4577-4580, 1996.

Zheng J, Shen W, He DZZ, Long KB, Madison LD, Dallos P. Prestin is the motor protein of cochlear outer hair cells. Nature 405: 149-155, 2000.

Zheng J, Long KB, Shen W, Madison LD, Dallos P. Prestin topology: localization of protein epitopes in relation to the plasma membrane. NeuroReport 12:1929-1935, 2001.

Ziegler U, Vinckier A, Kernen P, Zeisel D, Biber J, Semenza G, Murer H, Groscurth P. Preparation of basal cell membranes for scanning probe microscopy. FEBS Lett. 436:179-184, 1998. 